\title{
LAGRANGE INTERPOLATION FOR NATURAL COLOUR IMAGE DEMOSAICING
}

\author{
Kannan E.P \\ Department of Electronics of Communication Engineering, \\ Arunachala College of Engineering for Women, \\ Manavilai, Vellichanthai, Tamilnadu, India \\ epkannanece@gmail.com \\ Chithra T.V \\ Department of Electronics of Communication Engineering, \\ Arunachala College of Engineering for Women, \\ Manavilai, Vellichanthai, Tamilnadu, India \\ chithratvakul@gmail.com
}

Submitted: Jul, 15, 2021 Revised: Sep, 28, 2021 Accepted: Oct, 10, 2021

\begin{abstract}
The quality of the digital image of the camera is determined by the colour demosaicing. The size and cost of the camera is reduced by a single sensor to capture the image which uses a Bayer Colour Filter Array (CFA). The captured image by the camera is the CFA image and the missing components of Red $(R)$, Green $(G)$, and Blue $(B)$. The proposed system uses Lagrange interpolation in both directions to find the missing components of $G$. To get the whole $G$ image, Inter Channel Correlation (ICC) is utilized. Then Colour different interpolation is employed for the reconstruction of $R$ and $B$ image. A fine-tuning step is designed to improve the quality. Natural images which are obtained from Kodak and IMAX are used for performance analysis. The qualities of the reconstructed images are validated in terms of Peak Signal to Noise Ratio (PSNR). The comparative analysis with the state-of-the-art approaches in the literature shows that the Lagrange interpolation provides better quality than others.
\end{abstract}

Keywords: Interpolation, Lagrange interpolation, Bayer, Inter channel correlation, colour filter array, colour demosaicing.

\section{INTRODUCTION}

A single sensor array called CFA is used to capture the colours such as $R$, $G$, and $B$ in the scene. Bayer [1] introduced a CFA pattern in 1976 is the most commonly used CFA. In [2], the $G$ signal is sub-sampled at a higher rate due to fact that the $G$ is more sensitive to human visual system than the $B$ and $R$ signals. A demosaicing approach can estimate the missing components and construct the full colour image efficiently. In earlier systems, interpolation approaches such as bilinear [3], nearest neighbor [4] and cubic spline [5] are simply applied to all channels independently. They can interpolate missing signals in the smooth regions very well, but produce zippering effects in the high frequency regions.

The ICC is exploited by assuming that the hue of an image is slowly varying [6-8]. There are many algorithms proposed for demosaicing the Bayer pattern; however, only the latest algorithms are reviewed briefly in this section. In [9], alternating projection based approach is discussed for colour demosaicing. The initial estimation of three channels uses the method in [10] and is then decomposed into four sub-bands using 2D separable filters. The high frequency information in the $G$ channel is used for updating the $R$ and $B$ channel's high 
frequency information. The full colour image reconstruction is obtained by vertical and horizontal direction interpolation [2], which leads two candidates for each pixel. The selection is made by exploiting local homogeneity of the image in CIE Lab colour space and spatial averaging technique is used to cancel the colour artifacts.

In [11], directional linear minimum mean square-error estimation is described. The chromatic components of $G-R$ and $G-B$ images are filtered by the aforementioned algorithm. The filtered signal is used for missing $G$ signals estimation in both directions, and the obtained estimates are fused. Eventually, the resultant $G$ channel is used to estimate the $B$ and $R$ channel. In [12], the successive approximation is discussed which refines the missing data based on colour differences [12] at each iteration. Also, the zipper effect is removed by a spatially adaptive stopping criterion.

Directional filtering is discussed in [13] for colour channel interpolation with a posteriori decision. Edge directed interpolation approach is considered for $G$ channel interpolation. The obtained $G$ channel information is utilized for estimating $R$ and $B$ channel using bilinear interpolation. The Local Polynomial Approximation (LPA) approach in [14] considers the demosaicing approach as a de-noising problem similar to the approach in [11]. Instead of using fix-length filter, the directional anisotropic scale-adaptive de-noising method is used for denoising. In [13], self similarity driven based demosaicing approach is described. Non local means is adopted along with SSD for robust and noise free colour channel interpolation.

Low complexity colour interpolation scheme discussed in [16] is based on Integrated Gradient (IG). The combination of gradients in both colour intensity and colour difference domain are computed as IG which gives sufficient information to select the interpolation direction. In [17], Luma-chroma demultiplexing is described for Bayer demosaicing. In [17], the nonlocal adaptive thresholding is employed for the estimation of $\mathrm{G}$ channel by non local means filtering in [18].

Stencil based colour demosaicing approach is described in [19]. Contour stencils are applied for every mosaicked pixel. To estimate the true contour orientation, stencil that has smallest variation is selected. The work described in [20] focuses residual interpolation for image demosaicing. Instead of using colour differences for $R$ and $B$ channels, the residuals between the observed signals and the up-sampled data by guided filter. In [21], the residuals are computed using the Laplacian energy minimization approach and the observed data. In this paper, an approach for demosaicing the natural colour images based on Lagrange interpolation is proposed.

\section{METHODS AND MATERIALS}

The proposed demosaicing approach is shown in Figure 1. First, Lagrange formula of interpolation is applied to the green channel along row wise (horizontal direction) and column wise (vertical direction) independently. Second, the ICC is exploited to obtain the $G$ channel using interpolated $G$ and the original $B$ - $R$ values. In the next step, colour difference interpolation is employed to obtain the $R$ - $B$ values. Finally, a fine-tuning step is adapted to the interpolated channels to obtain a full resolution image. 


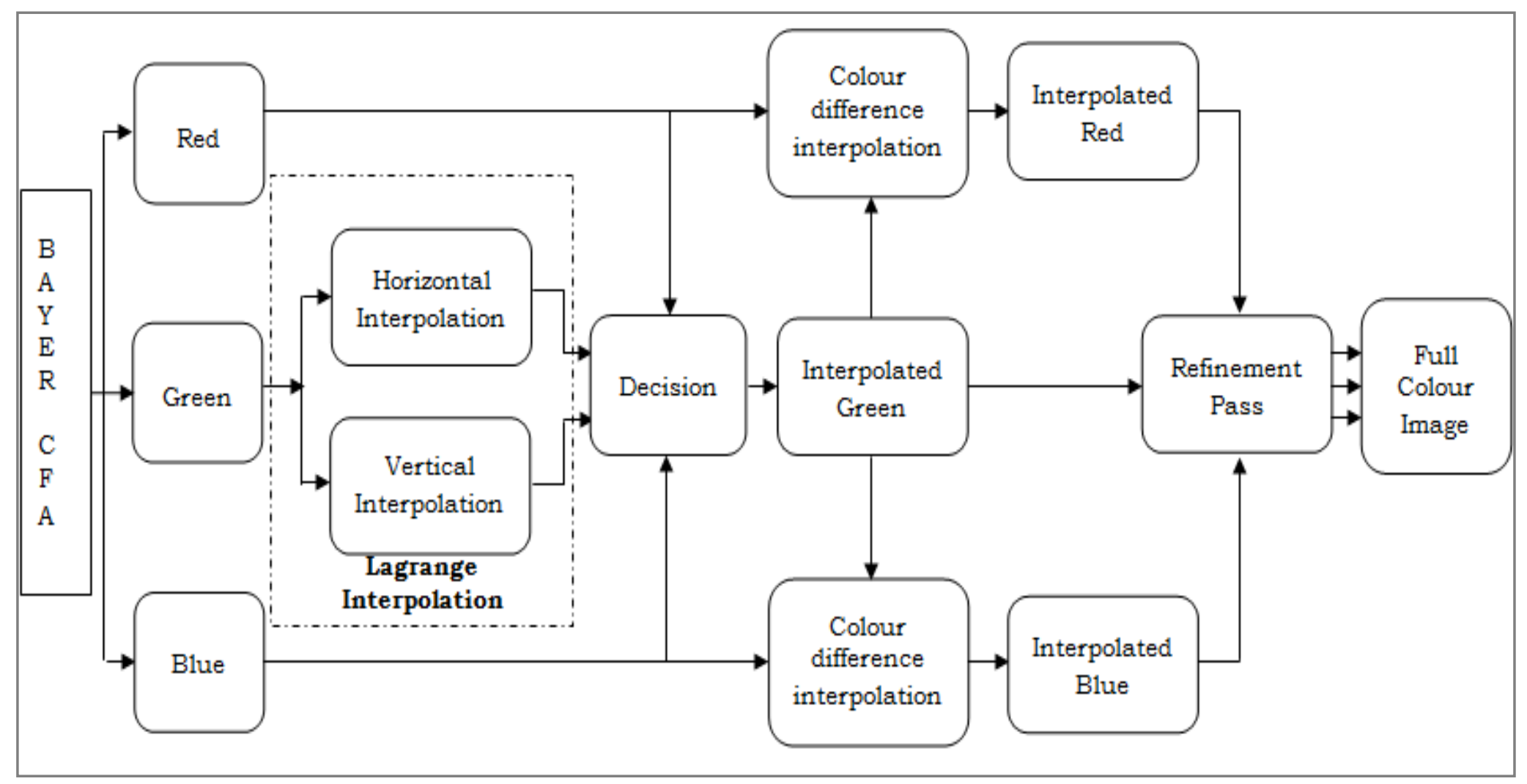

Fig. 1 Proposed colour demosaicing system 


\section{A. Lagrange Interpolation for $\mathrm{G}$}

The first step is the interpolation of $G$ channel. Among the $R, G$ and $B$ signals in the Bayer pattern, only the $G$ signal is sub-sampled by 2 in each row or column. Hence, almost all demosaicing approaches starts with interpolating $G$ signal first and then the remaining signals. To interpolate the $G$ signal, Lagrange formula of interpolation [22] is analyzed. The proposed $G$ channel interpolation is shown in Figure 2.

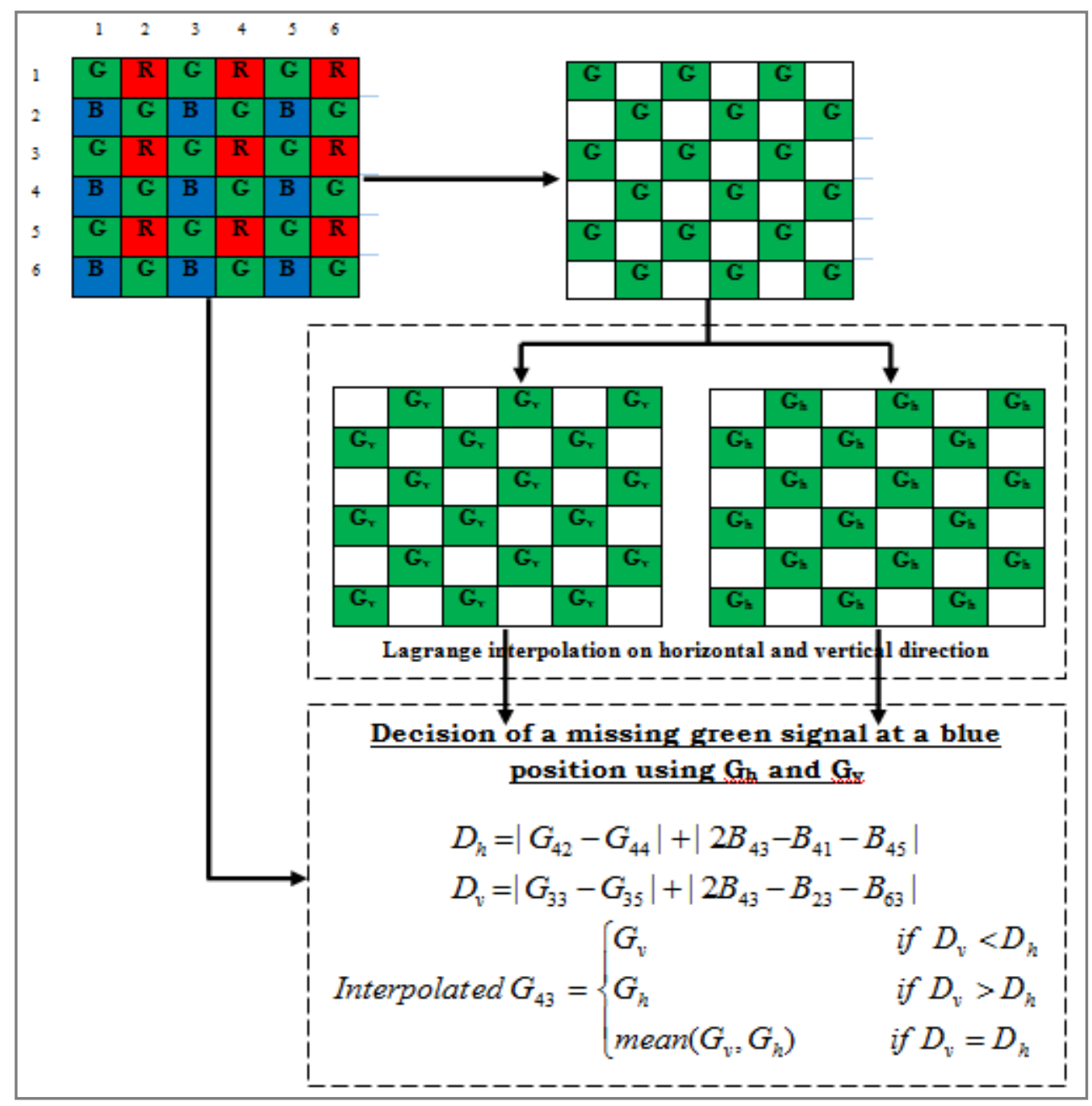

Fig. 2 G channel interpolation

In the proposed approach, Lagrange interpolation is applied on the available Bayer $G$ signals both row wise and column wise independently. It produces horizontal and vertical interpolated full $G$ channel images. In the Bayer pattern, $R$ or $B$ signal is available at the missing $G$ signal. Hence, to select an interpolation direction with least variation, a decision module is designed using the gradient of $R$ or $B$ signals. The gradient at the missing $G$ signal is estimated by the $2^{\text {nd }}$ order derivatives of $R$ or $B$ signals as ICC measure. The more information about gradient estimation can be found in [15 and 10] and is summarized at a B 
position in Figure 2 also. The same steps can be repeated for taking decision on a missing $G$ signal on $R$ position.

\section{B. R-B Channel Interpolation}

As the sub-sampling factor of $B$ and $R$ channels is 4 , it is not possible to obtain the interpolated horizontal and vertical signals using the original $B$ and $R$ signals. Hence a different approach is used for interpolating $B$ and $R$ channels. It is observed from the literature survey that mainly the colour difference interpolation is used for estimating the missing R-B signals. Figure 4 shows the colour difference interpolation used for reconstructing missing $R$ signals. In bilinear interpolation the missing $B$ and $R$ signals are computed by the average of their corresponding two adjacent signals. Instead of using $B$ and $R$ images directly, the colour differences are employed using the interpolated $G$ channel. The same steps in Figure 3 can be repeated for reconstructing missing $B$ signals.

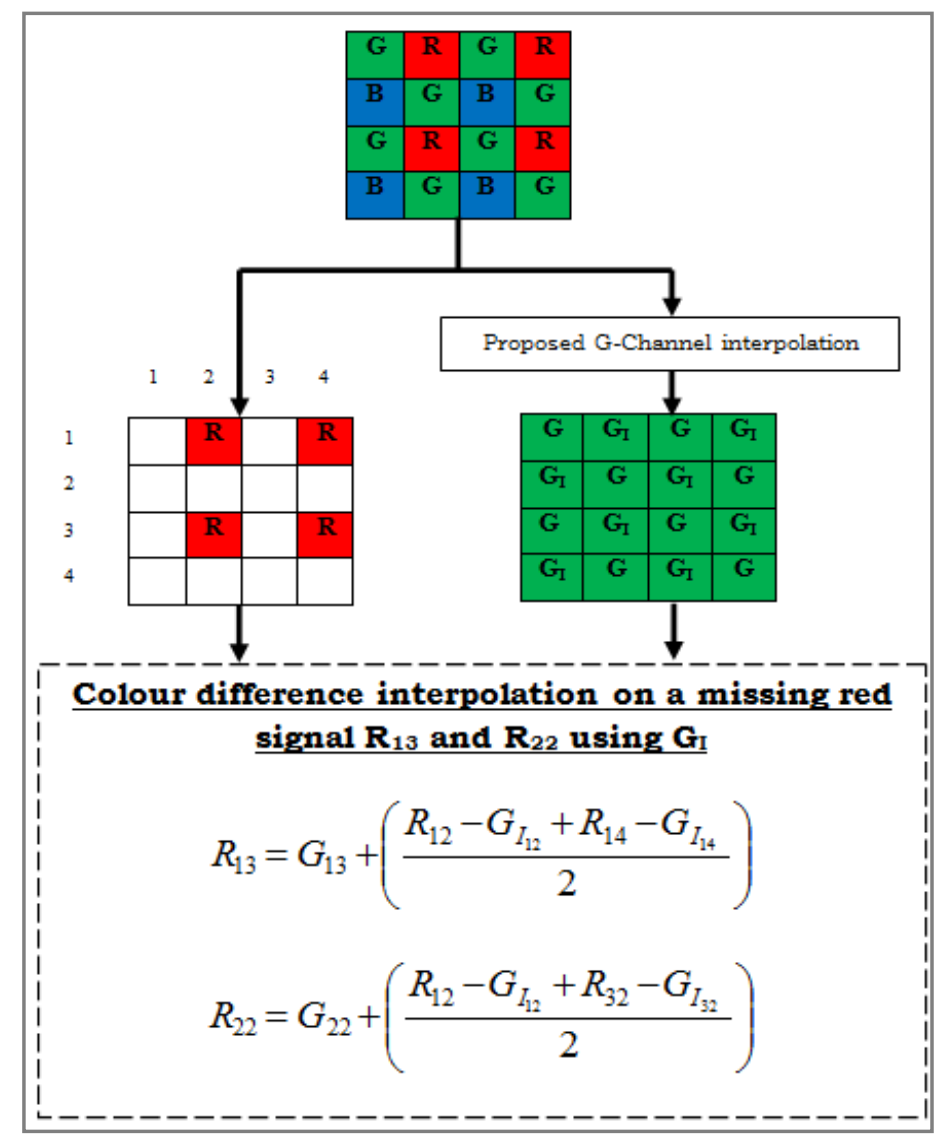

Fig. 3 Colour difference interpolation used for reconstructing missing $R$ signals.

\section{Fine-tuning Step}

This is the final process of this approach which further improves the quality of the images. The demosaicing approaches based on interpolation may contain artifacts which is also known as zipper effect. Usually, the high frequency components of an image contain artifacts that are needed to remove. In order to 
remove colour artifacts, most of the research concentrates on the inter channel correlation of high frequencies and known Bayer data. Some particular artifacts removal methods have been investigated in [9,12 and 13]. As the results in [5] provides better performance than others in the literature, the same approach is used to fine-tune the obtained interpolated signals. A low pass filter with $3 \times 3$ kernel $\left[\begin{array}{lll}1 & 1 & 1\end{array}\right] / 3$ is used to extract the low frequency components from each channel. Then, these components are subtracted from the original components. The obtained high frequency components by the interpolation signals are replaced by the original Bayer's high frequency components.

\section{RESULTS AND DISCUSSIONS}

This section discusses the performances of the proposed demosaicing algorithm using Lagrange interpolation. To analyze the system's performances, natural images from IMAX [23] (tiff images) and Kodak [24] (bitmap images) datasets are utilized. The former database has 18 images with 500x500 pixels whereas Kodak database has 12 images with $768 \times 512$ pixels. All images in these databases are shown in Figures 4 and 5.

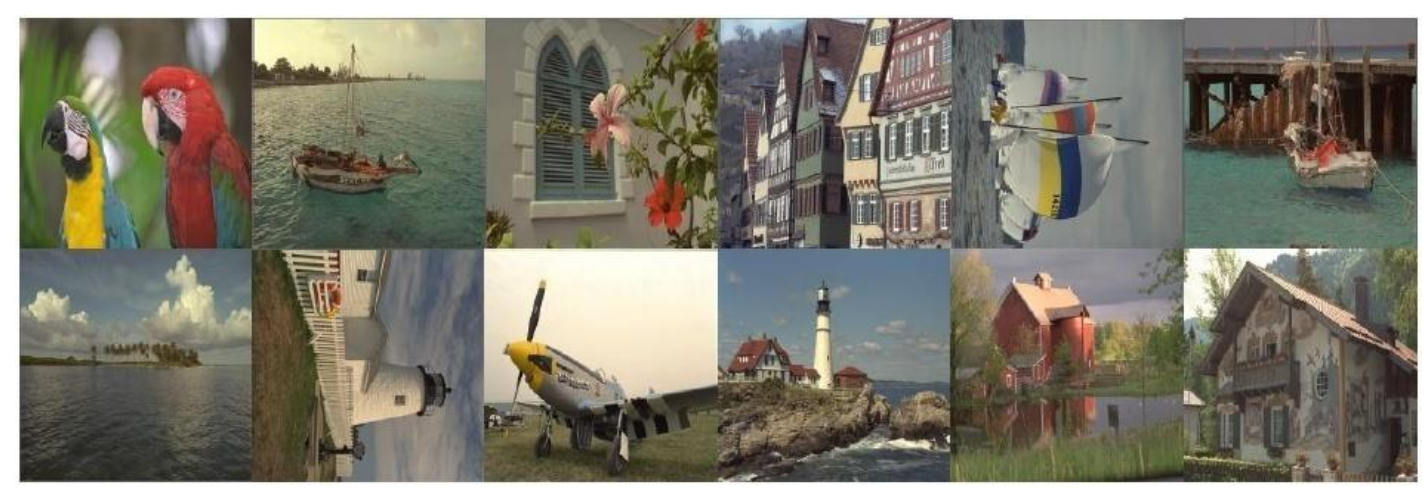

Fig. 4 Kodak database

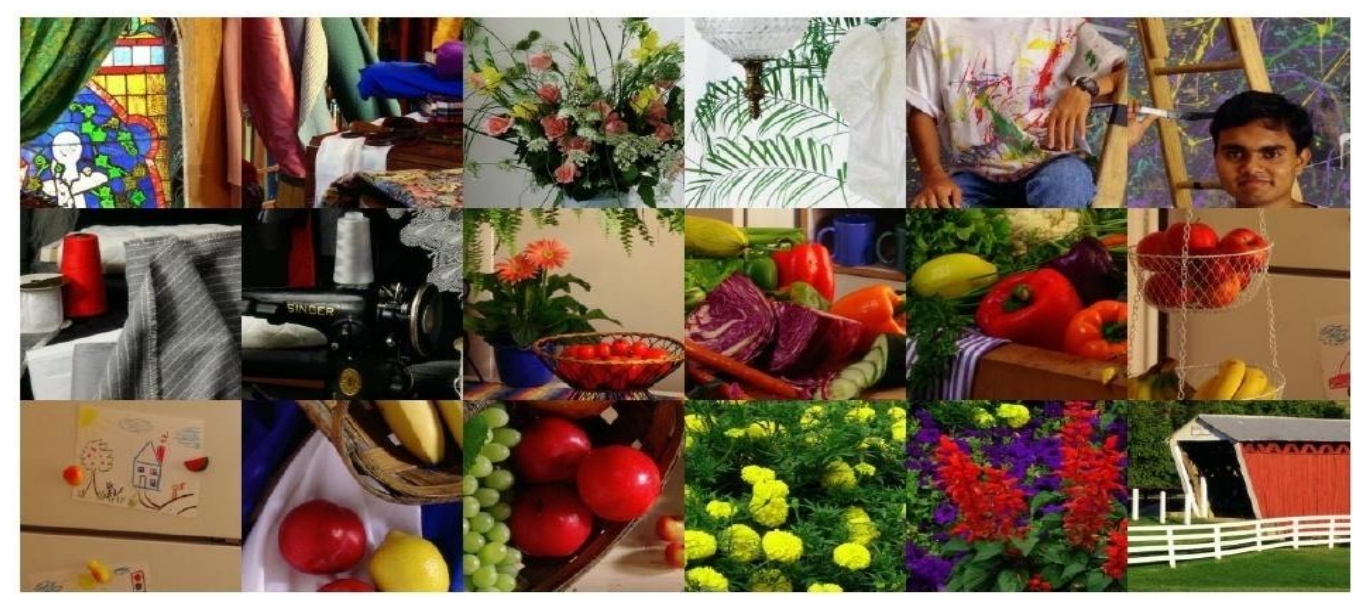

Fig. 5 IMAX database 
The reconstructed images by different algorithms are shown in Figures 6 and 7. To visualize the performances of the systems, only a cropped portion of the image is shown for all algorithms. Also, the obtained PSNRs for 5 samples from each database are shown in Tables 1 and 2 for Kodak and IMAX respectively. The PSNR is estimated from the original natural images in the database and the reconstructed from the algorithms.

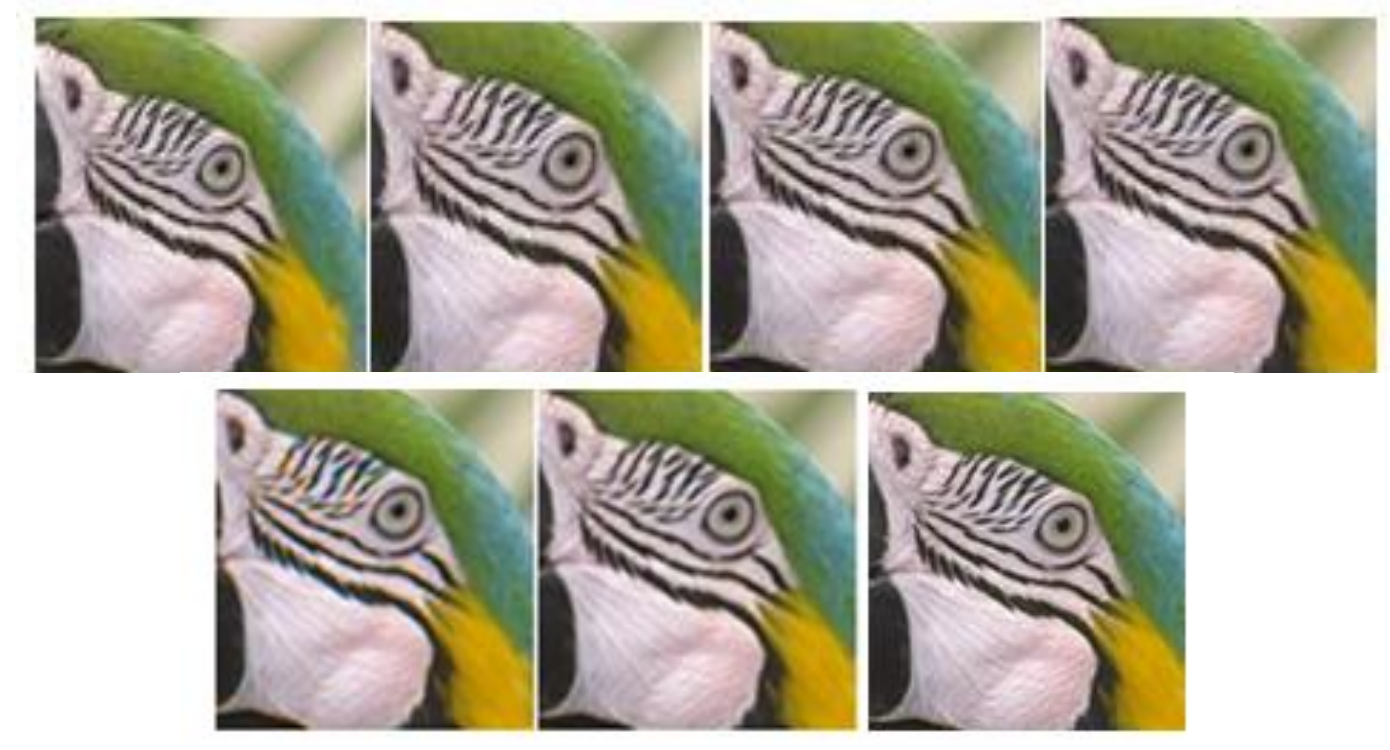

Fig. 3 Performance of a sample in IMAX database; Outputs from [11], [16], [18], [20], [21] and the proposed system (From top to bottom and right to left)
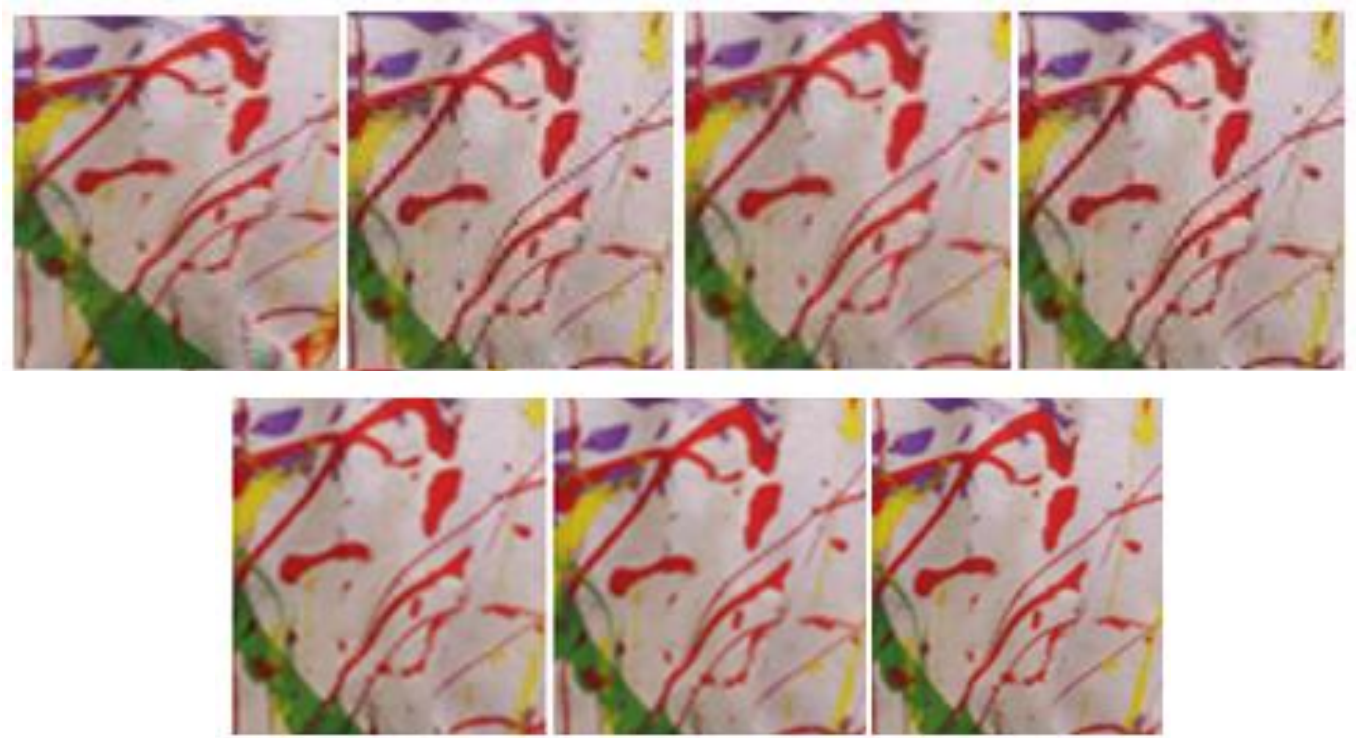

Fig. 3 Performance of a sample in Kodak database; Outputs from [1 1], [16], [18], [20], [21] and the proposed system (From top to bottom and right to left) 
TABLE. 1 Obtained PSNRs on Kodak database

\begin{tabular}{|c|c|c|c|c|c|c|c|}
\hline \multirow{2}{*}{ \#image } & \multirow{2}{*}{ Channel } & \multicolumn{7}{|c|}{ PSNR } \\
\cline { 3 - 8 } & & {$[\mathbf{1 1 ]}$} & {$[\mathbf{1 6}]$} & {$[\mathbf{1 8 ]}$} & {$[\mathbf{2 0 ]}$} & {$[\mathbf{2 1 ]}$} & $\begin{array}{c}\text { Proposed } \\
\text { system }\end{array}$ \\
\hline \multirow{3}{*}{$\mathbf{1}$} & $\mathbf{R}$ & 42.758 & 43.228 & 43.758 & 42.093 & 42.411 & 47.636 \\
\cline { 2 - 8 } & $\mathbf{G}$ & 44.423 & 44.891 & 45.423 & 44.749 & 44.592 & 43.284 \\
\cline { 2 - 8 } & $\mathbf{B}$ & 43.187 & 43.559 & 44.187 & 42.38 & 43.357 & 40.852 \\
\hline \multirow{3}{*}{$\mathbf{2}$} & $\mathbf{R}$ & 39.065 & 39.586 & 40.065 & 39.328 & 41.2 & 46.818 \\
\cline { 2 - 8 } & $\mathbf{G}$ & 41.253 & 41.564 & 42.253 & 41.421 & 40.446 & 43.284 \\
\cline { 2 - 8 } & $\mathbf{B}$ & 39.355 & 38.395 & 39.355 & 38.662 & 40.96 & 40.852 \\
\hline \multirow{3}{*}{$\mathbf{3}$} & $\mathbf{R}$ & 41.457 & 43.062 & 43.457 & 42.446 & 43.339 & 47.182 \\
\cline { 2 - 8 } & $\mathbf{G}$ & 43.259 & 45.843 & 46.259 & 45.791 & 45.944 & 43.821 \\
\cline { 2 - 8 } & $\mathbf{B}$ & 42.407 & 41.53 & 42.407 & 41.579 & 42.374 & 41.116 \\
\hline \multirow{3}{*}{$\mathbf{R}$} & $\mathbf{R}$ & 35.242 & 34.407 & 35.242 & 34.257 & 35.331 & 35.614 \\
\cline { 2 - 8 } & $\mathbf{G}$ & 36.134 & 37.425 & 38.134 & 37.316 & 38.942 & 43.658 \\
\cline { 2 - 8 } & $\mathbf{B}$ & 35.251 & 34.422 & 35.251 & 34.344 & 35.379 & 36.574 \\
\hline \multirow{3}{*}{$\mathbf{5}$} & $\mathbf{R}$ & 42.217 & 42.846 & 43.217 & 42.634 & 42.958 & 43.357 \\
\cline { 2 - 8 } & $\mathbf{G}$ & 42.757 & 45.252 & 45.757 & 45.115 & 45.543 & 43.284 \\
\cline { 2 - 8 } & $\mathbf{B}$ & 42.409 & 41.52 & 42.409 & 40.876 & 41.985 & 40.992 \\
\hline
\end{tabular}

It is observed from Table 1 that the proposed demosaicing approach for the Kodak database outperforms the other state-of-art approaches. The proposed demosaicing approach gives better PSNR for $G$. In $R$ - $B$ channel of IMAX database the proposed demosaicing approach and [18] yields better PSNR than all other algorithms. It is also observed from Table 2 that the proposed approach for the IMAX database outperforms the other state-of-art approaches.

TABLE. 2 Obtained PSNRS on IMX database

\begin{tabular}{|c|c|c|c|c|c|c|c|}
\hline \multirow{2}{*}{ \#image } & \multirow{2}{*}{ Channe1 } & \multicolumn{6}{|c|}{ PSNR } \\
\cline { 2 - 8 } & & {$[\mathbf{1 1 ]}$} & {$[\mathbf{1 6}]$} & {$[\mathbf{1 8 ]}$} & {$[\mathbf{2 0 ]}$} & {$[\mathbf{2 1 ]}$} & $\begin{array}{c}\text { Proposed } \\
\text { system }\end{array}$ \\
\hline \multirow{3}{*}{$\mathbf{1}$} & $\mathbf{R}$ & 26.982 & 29.489 & 34.012 & 29.162 & 30.012 & 34.669 \\
\cline { 2 - 8 } & $\mathbf{G}$ & 30.982 & 32.436 & 37.627 & 32.373 & 32.614 & 39.269 \\
\cline { 2 - 8 } & $\mathbf{B}$ & 22.893 & 27.45 & 31.417 & 26.981 & 27.648 & 33.307 \\
\hline \multirow{3}{*}{$\mathbf{2}$} & $\mathbf{R}$ & 33.482 & 34.853 & 35.683 & 34.479 & 35.011 & 34.179 \\
\cline { 2 - 8 } & $\mathbf{G}$ & 35.453 & 39.446 & 38.841 & 39.436 & 39.413 & 36.785 \\
\cline { 2 - 8 } & $\mathbf{B}$ & 30.232 & 33.296 & 32.557 & 33.21 & 33.28 & 31.967 \\
\hline \multirow{3}{*}{$\mathbf{3}$} & $\mathbf{R}$ & 32.562 & 34.255 & 35.996 & 34.041 & 34.863 & 38.304 \\
\cline { 2 - 8 } & $\mathbf{G}$ & 35.232 & 36.869 & 37.827 & 36.754 & 37.653 & 41.345 \\
\cline { 2 - 8 } & $\mathbf{B}$ & 19.312 & 31.293 & 31.349 & 31.738 & 32.76 & 35.352 \\
\hline \multirow{3}{*}{$\mathbf{4}$} & $\mathbf{R}$ & 34.972 & 37.274 & 35.784 & 38.304 & 38.265 & 36.895 \\
\cline { 2 - 8 } & $\mathbf{G}$ & 35.224 & 42.292 & 37.484 & 42.137 & 42.259 & 37.864 \\
\cline { 2 - 8 } & $\mathbf{B}$ & 33.453 & 33.895 & 30.771 & 35.527 & 35.602 & 30.85 \\
\hline \multirow{3}{*}{$\mathbf{5}$} & $\mathbf{R}$ & 30.642 & 37.081 & 38.872 & 36.804 & 38.347 & 39.057 \\
\cline { 2 - 8 } & $\mathbf{G}$ & 32.424 & 38.012 & 37.69 & 37.858 & 39.15 & 41.831 \\
\cline { 2 - 8 } & $\mathbf{B}$ & 28.242 & 31.608 & 31.24 & 30.728 & 32.299 & 35.934 \\
\hline
\end{tabular}




\section{CONCLUSION}

In this paper, an efficient demosaicing approach based on Lagrange formula of interpolation is presented. The reconstruction of green channel uses Lagrange formula of interpolation. The inter-channel correlation along with colour difference interpolation is used for red and blue channel. Before combining the colour channels, a refinement pass is performed for further improvement. Many experiments on two databases, IMAX and Kodak, are carried out. Experimental results are validated in terms of PSNR and the comparative analysis with the state-of-the-art approaches in the literature. It is concluded that the Lagrange formula of interpolation provides better quality than others.

Funding Statement: The authors received no specific funding for this study.

Conflicts of Interest: The authors declare that they have no conflicts of interest to report regarding the present study.

\section{REFERENCES}

[1]. $\quad$ B. E. Bayer, "Color Imaging Array”, U.S. Patent 3971 065, 1976.

[2]. K. Hirakawa and T. W. Parks, "Adaptive homogeneity-directed demosaicing algorithm", IEEE Transaction on Image Processing, Vol. 14, No. 3, 2005, pp. 360-369.

[3]. Hou, H. S and Andrews, H, "Cubic splines for image interpolation and digital filtering", IEEE Transaction on Acoustics Speech, Signal Process, Vol. ASSP-26, No. 3, 1978, pp. 508-517.

[4]. J. E. Adams, "Intersections between color plane interpolation and other image processing functions in electronic photography", Proceedings of the SPIE, Vol. 2416, 1995, pp. 144-151.

[5]. P. Longere, X. Zhang, P. B. Delahunt, and D. H. Brainard, "Perceptual assessment of demosaicing algorithm performance", Proceeding of the IEEE, Vol. 90, 2002, pp. 123-132.

[6]. R. Kimmel, "Demosaicing: Image reconstruction from CCD samples", IEEE Transaction on Image Processing, Vol. 8, No. 6, pp. 1221-1228, 1999.

[7]. D. R. Cok, "Reconstruction of CCD images using template matching", in Proceeding of IS\&T Annual Conference., Vol. 2, May 1994, pp. 380-385.

[8]. J. A. Weldy, "Optimized design for a single-sensor color electronic camera system", Proceeding of the SPIE, Vol. 1071, 1988, pp. 300-307.

[9]. B. K. Gunturk, Y. Altunbasak, and R. M. Mersereau, "Color plane interpolation using alternating projections", IEEE Transaction on Image Processing, Vol. 11, No. 9, 2002, pp. 997-1013.

[10]. J. Hamilton Jr and J. Adams Jr, "Adaptive Color Plane Interpolation in Single Sensor Color Electronic Camera”, U.S. Patent 5 629 734, 1997.

[11]. L. Zhang and X. Wu, "Color demosaicking via directional linear minimum mean square-error estimation", IEEE Transaction on Image Processing, Vol. 14, No. 12, 2005, pp. 2167-2178.

[12]. X. Li, "Demosaicing by successive approximation", IEEE Transaction on Image Processing, Vol. 14, No. 3, pp. 370-379, 2005. 
[13]. D. Menon, S. Andriani, and G. Calvagno, "Demosaicing with directional filtering and a posteriori decision", IEEE Transaction on Image Processing, Vol. 16, No. 1, 2007, pp. 132-141.

[14]. D. Paliy, V. Katkovnik, R. Bilcu, S. Alenius, and K. Egiazarian, "Spatially adaptive color filter array interpolation for noiseless and noisy data", International Journal of Imaging Systems and Technology, Vol. 17, No. 3, 2007, pp. 105-122.

[15]. A. Buades, B. Coll, J. M. Morel, and C. Sbert, "Self-similarity driven color demosaicking", IEEE Transaction on Image Processing, Vol. 18, No. 6, 2009, pp. 1192-1202.

[16]. K. H. Chung and Y. H. Chan, "Low-complexity color demosaicing algorithm based on integrated gradients", Journal of Electronic Imaging, Vol. 19, No. 2, 2010, pp. 021104-1-15.

[17]. B. Leung, G. Jeon, and E. Dubois, "Least-squares luma-chroma demultiplexing algorithm for Bayer demosaicking", IEEE Transaction on Image Processing, Vol. 20, No. 7, 2011, pp. 1885-1894.

[18]. L. Zhang, X. Wu, A. Buades, and X. Li, "Color demosaicking by local directional interpolation and nonlocal adaptive thresholding", Journal of Electronic Imaging, Vol. 20, No. 2, 2011, pp.023016-1-16.

[19]. P. Getreuer, "Image demosaicking with contour stencils", Image Processing on Line, Vol. 2, 2012, pp. 22-34.

[20]. D. Kiku, Y. Monno, M. Tanaka, and M. Okutomi, "Residual interpolation for color image demosaicking", Proceedings of IEEE International Conference on Image Processing, 2013, pp. 2304-2308.

[21]. D. Kiku, Y. Monno, M. Tanaka, and M. Okutomi, "Minimized-Laplacian residual interpolation for color image demosaicking", Proceedings of the SPIE, Vol. 9023, 2014, pp. 90230L-1-8.

[22]. E. Meijering, "A chronology of interpolation: from ancient astronomy to modern signal and image processing," Proceedings of the IEEE, Vol. 90, No. 3, 2002, pp. 319-342.

[23]. IMAX database: www4.comp.polyu.edu.hk/ cslzhang/CDM_Dataset.htm

[24]. Kodak color image dataset: http://rOk.us/graphics/kodak/. 